\title{
wissen kompakt „Dentale Biomaterialien“
}

\begin{abstract}
Dentale Biomaterialien werden zwar täglich in allen zahnmedizinischen Fachbereichen verwendet, doch sind Angebot und Neuentwicklungen kaum mehr zu überblicken. Die November-Ausgabe von wissen kompakt hilft Ihnen hier den Überblick zu bewahren. Neuestes zum Thema „Dentale Biomaterialien“ liegt dann für Sie bereit, um gut informiert das Jahr zu beenden.
\end{abstract}

\section{Befestigungsmaterialien in der restaurativen Zahnheilkunde Dr. Wolfgang Bömicke, MSc| Heidelberg}

Die Aufgabe dentaler Befestigungsmaterialien besteht darin, indirekte Restaurationen oder Restaurationsbestandteile vorübergehend oder dauerhaft in der Mundhöhle zu verankern. Insbesondere Kunststoffzemente gewinnen zunehmend an Bedeutung, jedoch stehen Zementreste bei Implantatrekonstruktionen auch im Verdacht, Entzündungen auszulösen. Der Fortbildungsbeitrag gibt eine allgemeine Übersicht über die gängigen Befestigungsmaterialien sowie Hinweise zu deren Inhaltsstoffen, Eigenschaften und Anwendung.

\section{Vollkeramische Restaurationen}

Prof. Dr. Daniel Edelhoff' ${ }^{1}$

PD Dr. Michael Stimmelmayr ${ }^{2}$

Prof. Dr. Florian Beuer ${ }^{3}$

PD Dr. Jan-Frederik Güth' | 'München, ${ }^{2}$ Cham, ${ }^{3}$ Berlin

Die konstante Weiterentwicklung keramischer Werkstoffe in den letzten Jahrzehnten hat zu erheblichen Verbesserungen der mechanischen Eigenschaften geführt. Vollkeramische Materialien bieten daher in vielen Bereichen eine Alternative zu metallbasierten Lösungen. Allerdings hängt der Langzeiterfolg noch immer sehr stark von der korrekten Indikationsstellung und Materialauswahl ab. Der Beitrag beleuchtet daher die charakteristischen Eigenschaften von dentalen Keramiken und hilft Ihnen, die Vorund Nachteile verschiedener Keramiktypen einzuschätzen sowie indikationsabhängig eine Materialauswahl zu treffen.

\section{Faserverstärkte Komposite}

PD Dr. Cornelia Frese, Prof. Dr. Dr. Hans Jörg Staehle, Dr. Diana Wolff | Heidelberg

Faserverstärkte Komposite werden aufgrund ihrer sehr guten mechanischen Eigenschaften seit Längerem im zahnmedizinischen Bereich eingesetzt. Hierfür werden anorganische Fasern durch funktionelle Anbindung in eine organische Phase integriert. Der Beitrag fokussiert sich auf die materialspezifischen Unterschiede verschiedener Arten faserverstärkter Komposite und deren Auswirkungen auf den klinischen Einsatz, um Ihnen einen Überblick zu biologischer Verträglichkeit, verschiedenen Techniken und klinischem Vorgehen zu vermitteln.

\section{Membrane und Membrantechniken}

\section{Dr. Dr. Thomas Ziebart | Mainz}

In der Implantologie und Parodontologie dienen Membranen und die unterschiedlichen Operationstechniken im Rahmen einer gesteuerten Geweberegeneration zur gezielten Regeneration von Hart- und Weichgewebe. Da der chirurgisch tätige Zahnarzt jedoch mit einer Fülle an unterschiedlichen Membra- nen konfrontiert wird, widmet sich der Fortbildungsbeitrag diesem Thema und beleuchtet die aktuellen Membrantypen sowie Verarbeitungstechniken, damit Sie diese gezielt für die tägliche Arbeit einsetzen können.

Die aktuelle Online-Ausgabe von wissen kompakt finden Sie unter www.springerzahnmedizin.de/wissen-kompakt

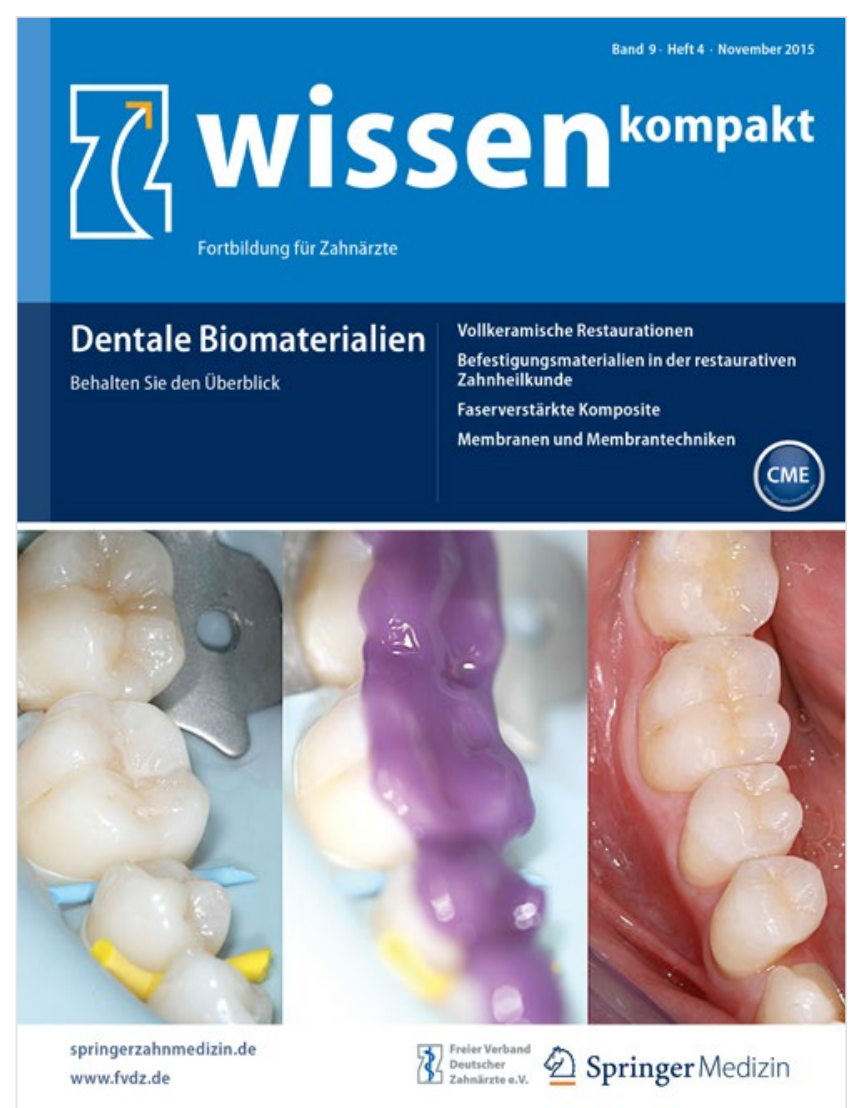

